\title{
Sinhogarismo y jóvenes extranjeros en Bilbao: la atención en un contexto de desbordamiento múltiple y secuencial de los recursos
}

\section{Gorka Moreno Márquez}

Ikuspegi-Observatorio Vasco de Inmigración

Departamento Sociología y Trabajo Social

Universidad del País Vasco/Euskal Herriko Unibertsitatea (UPV/EHU)

gorka.moreno@ehu.eus

\section{Iraide Fernández Aragón}

Ikuspegi-Observatorio Vasco de Inmigración

Departamento Sociología y Trabajo Social

Universidad del País Vasco/Euskal Herriko Unibertsitatea (UPV/EHU)

iraide.fernandez@ehu.eus

Giza desberdintasunak soziologia ikerketaren oinarri bat dira. Etxegabetasuna fenomeno honen erakusle nagusia izan arren, giza ikerketa ikuspuntutik gutxien landutako esparru bat da duen aztertzekonplexutasunagatik, besteak beste. Zaurgarritasun handienetako taldeen artean, azken urteetan EAEn (eta horren ondorioz, etxegabetasun presentzia handia dutenen artean), atzerritar gazteak ditugu. Pertsona hauek hainbeste arrisku faktoreengandik eragina daude: sare gabezia, formakuntza falta, bertoko hizkuntzaren ikasteko zailtasunak eta baliabide ekonomikoen falta, esaterako. Hau guztiak, bere biztanleriaren bolumen igoerarekin batera haien egoeraren inguruko diagnostiko bat egiteko beharra erakusten du. Horren ondorioz, artikulu honen helburu nagusia Bilbon etxegabetasun egoera bizi diren pertsonen egoera ezagutzea da, bereziki bakarrik dauden gazte atzerritarren egoera -JENA, gazteleraz- adituei egindako elkarrizketen bitartez.

\section{GAKO-HITZAK:}

Etxegabetasuna, gazteak, atzerritar, baliabide instituzionalak.
Las desigualdades sociales constituyen un objeto de estudio sociológico básico; sin embargo, el sinhogarismo como su manifestación más severa es uno de los ámbitos menos abordados desde la investigación social, en parte por su complejidad para ser estudiado. Entre los grupos sociales de mayor vulnerabilidad y, en consecuencia, con mayor presencia en el sinhogarismo, encontramos, en los últimos años en la Comunidad Autónoma Vasca a las personas más jóvenes de origen extranjero. Estas personas presentan una serie de factores de riesgo relacionados con la falta de red, de formación, el aprendizaje de la lengua local y otros económicos que, unidos al aumento de volumen, ponen de manifiesto la necesidad de realizar un diagnóstico de su situación. Este artículo tiene como objetivo principal conocer la realidad social de las personas sin hogar en Bilbao, poniendo especial atención en el nuevo perfil de los jóvenes extranjeros sin acompañamiento familiar -JENA-, a través de entrevistas en profundidad a personas expertas.

\section{Palabras ClaVe:}

Sinhogarismo, jóvenes, extranjeros, recursos institucionales. 


\section{Introducción}

Las desigualdades sociales han sido objeto de estudio para la disciplina sociológica desde su mismo nacimiento. De entre todas las manifestaciones urbanas de la desigualdad, las personas sin hogar representan la exclusión social más extrema; el término alude a un fenómeno social en el que se agrupan factores de carácter individual, pero, especialmente, factores de carácter estructural. En su origen se encuentran causas económicas, políticas y sociales que impiden el acceso a la vivienda y a un empleo y, como consecuencia, a la integración sociocomunitaria. Sin embargo, y a pesar de ser la manifestación más cruda y polarizada de la desigualdad, el sinhogarismo es uno de los ámbitos menos abordados desde la investigación social en España y Euskadi, especialmente si atendemos a la variable migratoria.

El sinhogarismo no es un fenómeno novedoso en nuestra sociedad; no obstante, los estudios más recientes sobre el tema detectan que el perfil de persona sin hogar presente en la calle y atendida por los recursos institucionales está variando y aumentando sensiblemente su volumen. Se trata de un fenómeno que tiende a concentrarse en las grandes urbes, en opinión de las personas expertas, porque es aquí donde se ofrece la mayor parte de los servicios y recursos para este colectivo, pero también a consecuencia de ello. En este sentido, el foco de esta investigación se centrará en Bilbao, el municipio que en anteriores estudios se revela como el lugar de residencia habitual para más personas sin hogar tanto para el territorio histórico de Bizkaia, como para el conjunto de la Comunidad Autónoma Vasca.

Así, este artículo tiene como objetivo describir la realidad social de las personas sin hogar en Bilbao, prestando especial atención a un perfil cuyo volumen ha aumentado en los últimos años, el de los jóvenes de origen extranjero, muchos de los cuales anteriormente formaron parte del colectivo de menores extranjeros sin acompañamiento familiar (menas). Para comprender esta compleja realidad, se ha consultado la opinión de las personas expertas en materia de sinhogarismo - a través de entrevistas en profundidad- para así poder conocer en detalle la situación y cómo ven estas personas el momento actual y el futuro a corto plazo.

Se han trabajado diferentes temas dentro de las entrevistas que se detallan en el presente artículo. En primer lugar, se profundiza en la cuantificación del colectivo, siendo conscientes de las dificultades que entraña esta cuestión por las características de las personas sin hogar, así como por la utilización de las entrevistas para hacer estimaciones de un posible número de personas sin hogar. En segundo lugar, el artículo se centra en la caracterización de las personas sin hogar y de los perfiles y diferencias que pueden encontrarse dentro del colectivo. Además, se centra en los llamados jóvenes extranjeros sin acompañamiento familiar (JESAF), estudiando las principales características de este grupo, así como su situación y sus principales características. Por último, se evalúan los recursos existentes y específicamente la coordinación interinstitucional y la cuestión competencial, que aparece como un elemento clave cuando se trabaja el sinhogarismo en el País Vasco.

\section{Objetivos y metodología}

En este apartado, y como contexto de la investigación, se realizará una descripción de la metodología empleada en este trabajo, cuyo objetivo general era conocer la realidad social de las personas sin hogar en Bilbao, con especial atención al nuevo perfil de los y las jóvenes de origen extranjero. Este objetivo general se concreta en algunos objetivos específicos:

- Conocer la opinión de los diferentes agentes expertos que participan y trabajan en el campo de trabajo del sinhogarismo.

- Describir el volumen y perfiles actuales del sinhogarismo en Bilbao.

- Conocer las necesidades actuales y las expectativas de futuro, a corto y medio plazo, de estas personas sin hogar.

- Detectar puntos de mejora en la cobertura de servicios.

- Subrayar los posibles desajustes que puedan darse entre necesidades del colectivo y servicios.

Para cumplir dicho objetivo general y con el fin de realizar un análisis de carácter explicativo se ha optado por una metodología cualitativa de análisis de contenido. En consecuencia, se empleará la técnica de entrevista en profundidad. Vallés (2003) marca una serie de ventajas en el empleo de esta técnica, por ejemplo su naturaleza abierta, que permite obtener gran cantidad de información rica en matices y facilita a la persona investigadora recabar su información de manera más directa y flexible, entre otras (Vallés, 2003: 55). Es, en consecuencia, la técnica ideal para completar los objetivos específicos propuestos.

En concreto, se han realizado doce entrevistas en profundidad a personas expertas. En esta investigación se ha planteado que aquellas personas que gestionan recursos, servicios o tienen contacto con personas sin hogar son las que consideraremos expertas. Así, se han realizado siete entrevistas a personal y dirección de organizaciones y entidades sociales y cinco a personal y dirección de instituciones clave (Diputación Foral de Bizkaia, Ayuntamiento de Bilbao, Servicio Municipal de Urgencias Sociales y Policía Municipal). Dichas entrevistas se realizaron entre los meses de febrero y marzo de 2018 y tuvieron una duración aproximada de dos horas.

Los resultados que se presentan en este artículo forman parte de un estudio más amplio en el que 
la mirada experta se completa con un sondeo cuantitativo al colectivo de personas sin hogary entrevistas en profundidad a los jóvenes de origen extranjero en situación de exclusión social, “Estudio sobre las personas sin hogar en Bilbao" (2018), realizado fruto de la colaboración entre Ayuntamiento de Bilbao y la Universidad del País Vasco/Euskal Herriko Unibertsitatea (UPV/EHU).

\section{Antecedentes y marco conceptual}

El estudio de la exclusión social suele realizarse desde el análisis de las diferencias económicas basadas en la falta o escasez de ingresos, entendiendo que este es el indicador básico que caracteriza dicha desigualdad. Sin embargo, los colectivos y sectores más vulnerables y excluidos socialmente presentan una multiplicidad de privaciones que hacen necesario un análisis de las desigualdades no solo económicas para comprender una realidad social compleja. Por tanto, no podemos hablar del colectivo de personas sin hogar sin antes detenernos en lo que se considera exclusión social.

El concepto de exclusión social comienza a utilizarse en el contexto europeo a finales de la década de los ochenta, en el marco de la lucha contra un nuevo tipo de pobreza de carácter multidimensional fruto de los cambios acelerados que la sociedad sufría en ese momento (Arriba, 2002). Sin embargo, no es hasta unos años después que se produce un cambio real en la conceptualización de la pobreza y comienza a utilizarse la exclusión social como concepto. Su uso, sin embargo, no está exento de controversia y heterogeneidad. Conviven múltiples interpretaciones del término en los debates políticos y académicos con otros términos referidos a procesos sociales similares: marginación, pobreza, underclass, etc. Las concepciones varían además en función del país, la disciplina académica y los colectivos a los que se refiere (Arriba, 2002). Sin embargo, podríamos definirlo en contraposición al término "pobreza económica” como una categoría que pretende considerar aspectos del fenómeno relacionados con su carácter multidimensional, relativo y cambiante. Precisamente el consenso social y académico en torno a la necesidad de considerar otros factores llevó a desterrar el concepto de pobreza (de corte economicista) por el de exclusión social, que incluye los tres ejes o dimensiones: económico, político (participación) y social (relacional) (Laparra y Perez, 2008). Es decir, una situación sufrida fruto de un proceso de acumulación de factores de vulnerabilidad (multidimensional) que afecta a una persona o grupo y que impide su integración social, laboral y/o comunitaria.

Tan importante como definir el concepto y las situaciones que genera es comprender su origen y causas. El abordaje explicativo de la exclusión social se ha realizado a menudo desde la teoría de la underclass (o "infraclase") desarrollada en el Reino Unido (Wilson, 1987). Myrdall afirmaba ya en el año 1962 que esta clase estaba compuesta por personas paradas de larga duración, subempleadas y aquellas personas que por sus características personales eran poco empleables, con una formación baja y, en definitiva, en los márgenes de la sociedad (Myrdall, 1962). El concepto de “underclass” está fuertemente ligado a las teorías sobre la desviación social desarrolladas en la década de los setenta que relacionan a determinados colectivos (entre los que se encuentran las personas sin hogar) con una conducta antisocial o, en otras palabras, que afirman que las personas excluidas lo son porque se niegan a seguir las normas establecidas y, debido a su degradación, no logran integrarse en las estructuras básicas de empleo y familia (Vilagrasa, 2000).

Esta teoría ha recibido numerosas críticas desde las perspectivas más progresistas, que rechazan el determinismo psicologista, próximos a la idea de cultura de la pobreza. Asimismo, es muy frecuente asimilar el concepto "underclass" a actitudes estigmatizadoras y, en consecuencia, discriminatorias (Fainstein, 1993). Estas perspectivas críticas surgen como alternativa para dar explicación a los procesos de exclusión social, haciendo énfasis en la negación de oportunidades de ciertos grupos estigmatizados. En este sentido, Harrington consideraba el aumento de la pobreza como una consecuencia directa del proceso de desindustrialización de Estados Unidos, que supuso un aumento de la tasa de paro que afecta principalmente a las capas de población menos formadas (Harrington, 1984). Desde esta visión de carácter estructural, otros autores más recientes como Castells o Subirats coinciden en la existencia de barreras o mecanismos que dificultan o impiden la integración social, es decir, aluden al componente estructural de la exclusión social, casi como parte inevitable de la lógica del sistema económico -capitalista neoliberal- actual (Castells, 2003; Subirats, 2004).

Incluidos en los denominados "grupos vulnerables" o "colectivos en riesgo de exclusión social" encontramos aquellos que sufren una exclusión social severa o se encuentran excluidos en todas las dimensiones anteriormente mencionadas: las personas sin hogar. Este grupo social, según la clasificación ETHOS realizada por la federación europea Feantsa ${ }^{1}$ (Feantsa, 2008), puede dividirse en:

- Sin techo (rooflessness).

- Sin vivienda (houselessness).

- Vivienda insegura (insecure housing).

- Vivienda inadecuada (inadequate housing).

El sinhogarismo más severo sería el de quienes viven literalmente a la calle, "sin techo", y carecen de un

${ }^{1}$ Fédération Européenne des Associations Nationales Travaillant avec les Sans-Abri. 
lugar para residir y, por tanto, no disponen de un espacio para la privacidad y las relaciones sociales. La escala de gravedad va descendiendo, por lo que las personas "sin vivienda" son aquellas personas alojadas temporalmente en un albergue o servicio municipal, pasando después a la "vivienda insegura" -temporal, por ejemplo, en casa de un conocidoy a la "vivienda inadecuada" - chabolismo, hacinamiento, etc.-.

Debe considerarse además que, dentro del colectivo de personas sin hogar que de por sí se enfrenta a una actitud estigmatizadora por parte del resto de la sociedad, las personas migrantes sin hogar se enfrentan a una doble estigmatización: por ser personas sin hogar y por ser personas migrantes. Esta estigmatización influye en la su autopercepción, autoestima, identidad y puede suponer un obstáculo más en su integración sociocomunitaria (Navarro, 2018).

A pesar de las dificultades en términos de operacionalización y trabajo de campo que implica el estudio del fenómeno del sinhogarismo, en la última década se han realizado algunas investigaciones en el ámbito vasco. Cabe destacar la realizada a demanda de la Diputación Foral de Bizkaia por Xabier Aierdi, Gorka Moreno, José Antonio Oleaga e Iratxe Herrero, que serviría como diagnóstico sociológico básico para posteriores investigaciones (Aierdi et al., 2009), entre las que se encuentra la que se presenta en este artículo. Entre otros resultados, cabe señalar la concentración de personas sin hogar y sin recursos en el municipio bilbaíno y la aparición de nuevos perfiles vinculados a la migración que en ese momento restaban peso por primera vez al perfil tradicional de persona sin hogar. Se detectaban ya dos perfiles: aquellas personas con problemas personales (vinculados a consumos), mayoritariamente autóctonas; $y$ aquellas que fundamentalmente tenían un problema de carácter residencial y económico, en el que destacaban los jóvenes magrebíes (Aierdi et al., 2009).

Asimismo, Miguel Ángel Navarro realizaba en el 2018 una investigación de corte cualitativo - "Relatos de la calle: Testimonios de migrantes sin hogar"- poniendo el foco en las personas migrantes y aunando los planteamientos de la sociología y la psicología. Entre sus conclusiones, destaca la necesidad de ubicar la problemática del sinhogarismo dentro de la estructura social y no como consecuencia de ella, es decir, evitar pensar en el colectivo como parte de un mundo paralelo (Navarro, 2018).

En esta misma línea, la asociación Bizitegi publicaba en 2019 un informe en el que centraba su atención en las mujeres sin hogar, un grupo históricamente invisibilizado pero que, como se observará en el análisis de resultados de este artículo, requiere de especial atención por su especificidad y riesgos adicionales. Este informe, a través del análisis cualitativo, muestra no solo la existencia y caracterización de los diferentes perfiles de mujer sin hogar, sino también que el escaso porcentaje de presencia en la calle y la falta de conciencia social han derivado en un desarrollo de recursos diferenciados casi nulo, optando por servicio mixtos que no responden a las demandas específicas de las mujeres (Bizitegi, 2019).

\section{Resultados}

Tal y como se ha planteado ya a lo largo del artículo, en este apartado analizaremos detenidamente las principales características y la situación de las personas sin hogar residentes en Bilbao, haciendo especial hincapié en el colectivo de los jóvenes extranjeros. Para ello, analizaremos cuestiones como la cuantificación del colectivo, los perfiles predominantes, la situación y problemática de los jóvenes extranjeros o las necesidades y recursos existentes.

\subsection{Estimación y cuantificación del colectivo}

En este apartado, y teniendo en cuenta la metodología utilizada, más allá de dar una cifra exacta o concreta sobre el número de personas sin hogar que residen en Bilbao, el objetivo es poder vislumbrar cuál es la tendencia y comparar la situación actual con otros momentos. Del mismo modo, es destacable que para la definición del colectivo hemos utilizado la clasificación ETHOS de Feantsa y, más concretamente, las dos categorías de mayor de vulnerabilidad, que serían las de aquellas personas que pernoctan en la calle o en centros habilitados para este colectivo.

De esta forma, un primer aspecto relevante a destacar es la percepción de un importante aumento del número de personas sin hogar en los últimos años, sobre todo a partir del 2017 y con más intensidad aún en el 2018.

En las últimas reuniones de Bestebi, la sensación de todos es que ha habido un aumento exponencial de gente [...]. Esa es la sensación de todas las organizaciones. (E 6)

A nivel de números estamos peor que hace dos años. En la calle contábamos cien y hemos llegado a contar doscientos donde antes contábamos cien. Eso es una realidad, es un dato objetivo. Está ahí. (E 8)

El aumento de cifras es considerable [...]. Este es el año en el que ha habido más número de atenciones en los últimos cinco [años]. En todos los datos es el que más. (E 7)

De esta forma, se detecta una pauta que a priori puede parecer contraintuitiva. Durante el periodo que comprende la recesión económica, el incremento de personas sin hogar es muy limitado e insignificante; 
y es, precisamente, en el contexto poscrisis, cuando aumenta.

La crisis afectó al conjunto de la población, pero creo que no terminó de traducirse en un aumento de la gente sin hogar. (E 5)

Quitando los últimos meses, los diferentes recuentos nos indican que la cifra no ha aumentado demasiado en los últimos años. (E 3)

Con respecto al aumento detectado, una primera característica es la ruptura con la estacionalidad de las magnitudes dentro del colectivo. Así, si en años anteriores se detectaba que en los meses menos fríos la cifra disminuía, en la actualidad se percibe que el número de personas sin hogar se mantiene estable a lo largo de todo el año.

Normalmente en verano se nota todos los años que hay mucha gente que se marcha. Pero este año no se ha marchado. (E 3 )

Esos flujos que se daban de verano a invierno, entendido de noviembre a abril, no se dieron. (E 8)

Estos cambios dentro del colectivo, tanto en la magnitud como en algunas de sus características, se deben en gran parte al aumento de las personas sin hogar jóvenes extranjeras no acompañadas -JENA-, que en gran parte provienen de recursos forales de atención a menores extranjeros no acompañados -menas-, del propio país de origen o de otras Comunidades Autónomas. De hecho, la llegada de menas y JENA se ralentizó y estancó durante el periodo de recesión y en los últimos años, en cambio, se detecta un relevante repunte.

\section{El fenómeno de los JENA y menas había descendido durante la crisis. (E 2) \\ Durante la crisis dejan de venir. (E 4)}

Entre 2010 y 2015 hay una bajada importante, desde 2015 a 2017 vuelve a haber un repunte. (E 9)

Lo que ha pasado es que han llegado jóvenes del Magreb, alguno subsahariano, pero sobre todo del Magreb. También está el tema de los menores institucionalizados, que cumplen 18 años y van directamente o van con el educador del Servicio Municipal de Urgencias Sociales. (E 3)

En definitiva, se vislumbra un escenario en el que se detecta un importante aumento del número de personas sin hogar, tanto utilizando recursos de pernoctación como durmiendo en la calle. Este aumento coincide con un escenario poscrisis y sobre todo está condicionado a la llegada de menas y JENA en estos últimos años, lo que ha hecho que el perfil de hombres jóvenes provenientes del Magreb haya aumentado considerablemente dentro del conjunto de las personas sin hogar, tal y como podremos observar en el siguiente apartado.
4.2. Principales perfiles entre las personas sin hogar

A grandes trazos, se mantienen los perfiles detectados anteriormente en otros estudios (Aierdi et al., 2009), caracterizados por dos grandes grupos: el compuesto por población autóctona con un alto deterioro de salud y vinculado a la figura del transeúnte de otras épocas, y el de personas jóvenes de origen magrebí, con un mejor estado de salud. En todo caso, lo que sí se detecta es un constante aumento del segundo de estos perfiles, en detrimento del primero.

Yo he visto que hay un alza de personas menores de treinta años. A lo largo de los años el montante solía ser el de hombres solos, más tipo carrilano, de mediana edad y con problemáticas añadidas. Que iba de albergue en albergue. Pero esto se ha quedado obsoleto ya, se ha quedado en el olvido. Ahora existen otras problemáticas asociadas.

Sobre todo de personas migrantes y jóvenes. (E 7)

En los recursos de alojamiento de urgencia va disminuyendo la población nacional con problemáticas de adicciones, fundamentalmente alcoholismo, aunque también otras adicciones. (E 9)

En referencia al sexo, sigue manteniéndose de forma muy palmaria el predominio de los hombres frente a las mujeres, aunque las personas entrevistadas son conscientes de que en algunos casos no se está consiguiendo llegar al colectivo de las mujeres en situación de exclusión social extrema, en gran parte vinculado a deterioros muy altos y/o situaciones de prostitución en muchos casos. Así, nos encontramos ante la invisibilización del fenómeno de las mujeres sin hogar, que queda escondido bajo otras situaciones o contextos.

Hay mujeres con un historial de prostitución. Con problemáticas de salud física y, en muchos casos, también de salud mental derivados del tipo de trato que han recibido. (E 9)

Tenemos muy claro que en el tema del sinhogarismo las mujeres pasan desapercibidas [...]. Antes de acabar en la calle acabas durmiendo con algún desgarramantas, por decirlo de alguna manera. Eso no lo vemos, no sale. En los albergues tenemos pocas mujeres en proporción a los hombres, pero entendemos que ahí hay una parte que no se visibiliza, que no se ve claramente y que hay que abordar. (E 8)

Otro perfil, también minoritario en términos cuantitativos pero con unas características especiales, es el de las familias, sobre todo por la inexistencia de recursos adecuados y el riesgo de la situación para menores; sobre todo se detectan familias monoparentales en tránsito o solicitantes de asilo.

Hay un perfil importante y preocupante, que son las familias, que muchas veces son 


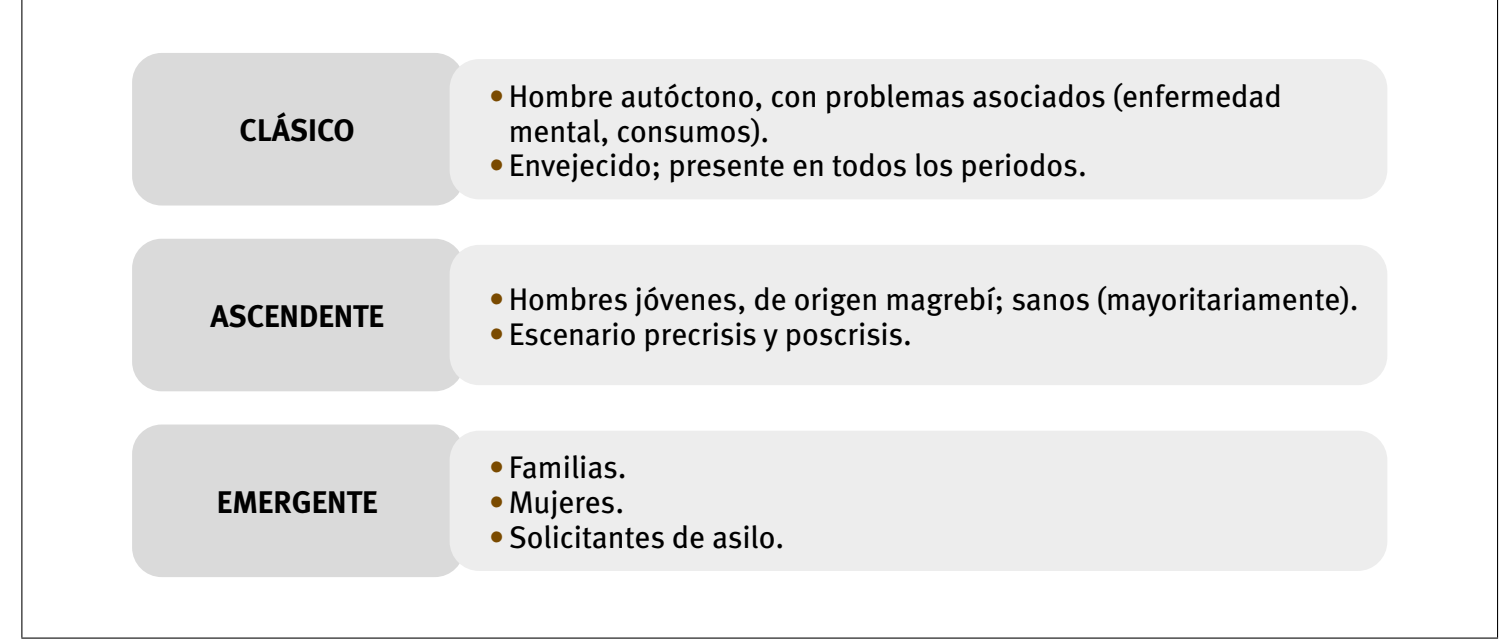

Fuente: Elaboración propia.

monoparentales, que están viajando para solicitar asilo o para solicitar ayuda porque huyen de sus países. (E 7)

En conclusión, podemos subrayar la existencia de varios perfiles dentro del colectivo de personas sin hogar, aunque su peso dentro de este sea muy desigual. En primer lugar, siendo el grupo más relevante y además en auge, nos encontramos con las personas sin hogar de origen extranjero y jóvenes, mayoritariamente magrebíes. En segundo lugar, y perdiendo fuerza a lo largo del tiempo, nos encontramos con las personas sin hogar autóctonas, con un amplio recorrido en el circuito de la exclusión social y un alto deterioro personal; es un colectivo cada vez más envejecido y cronificado. Por último, podríamos subrayar una serie de nuevos perfiles, que, aunque minoritarios, sí que entrañan un importante reto para la atención a las personas sin hogar, sobre todo por sus características y peculiaridades. Entre estos cabe destacar a las mujeres, las familias y las personas $\mathrm{y} / \mathrm{o}$ familias solicitantes de asilo.

\subsection{Los jóvenes extranjeros no acompañados (JENA)}

A lo largo de los puntos anteriores ya se ha mencionado la relevancia que ha tomado el colectivo de los jóvenes extranjeros dentro de las personas sin hogar, y más concretamente los procedentes del Magreb y especialmente de Marruecos. Aunque pueden encontrarse diferentes perfiles, las personas entrevistadas subrayan el aumento de exmenas que han sido tutelados hasta el cumplimiento de la mayoría de edad y que tras su salida de las instituciones forales se ven abocados a una situación de sinhogarismo al llegar a los dieciocho años.

Cada vez se ve más a los menores no acompañados que acaban su periodo de tutela en las diputaciones forales y que a los dieciocho cesan su proceso. (E 2)
La gran mayoría son los que salen de centro. (E 7)

Aunque también hay jóvenes que llegan habiendo cumplido los dieciocho años y acceden directamente a los servicios dispuestos para las personas sin hogar, bien directamente desde su país de origen, habiendo pasado por algún Centro de Internamiento de Extranjeros (CIE) o incluso algún centro tutelado de otra comunidad autónoma.

Los recién llegados están viniendo directamente de su país. Han pasado por los CIE de Andalucía o han estado algunos días en Andalucía y se han venido para acá. (E 4)

Está la persona joven que en un momento dado viene de un centro de menores de otra comunidad y aquí no tiene acceso. Está también la persona joven que llega y no ha pasado por ningún centro, que viene directamente. (E 6)

Están viniendo también exmenas tutelados de otras comunidades, poquitos, pero alguno sí. (E 9)

Para explicar dicho aumento, las personas expertas subrayan diferentes factores, aunque el que aparece con más fuerza es la existencia de redes migratorias, que ofrecen información sobre el contexto y los recursos existentes y que juegan también a modo de acogida en la llegada, algo por otro lado muy común y habitual dentro de los flujos migratorios generales.

Y hay mucha gente que sale del país de origen con un papelito donde tiene apuntado Bilbao. [...] La mayoría vienen aquí porque es de lo que han oído hablar en el lugar de origen”. (E 9)

Con respecto a las características de este colectivo, tal y como ya se ha apuntado brevemente en un punto anterior, son jóvenes con un buen estado de salud en general y en su mayoría con una clara voluntad para formarse y seguir un itinerario de 
incorporación social adecuado, aun en contexto vitales realmente complejos y complicados.

Hay muy poca gente de esta [JENA], que esté como perdida. (E 5)

Yo creo que un porcentaje muy alto de los chavales aprovechan los recursos y tienen claro su proceso. (E 12)

Siguen yendo a clase y sabes que están en la calle, ves que son supervivientes. Están en la calle, van a cursos y además muchos narran procesos migratorios duros. (E 7)

En este itinerario de inserción toma un papel fundamental la formación, ya que se erige en el anclaje básico que da sentido a todo el proceso; pero, además, en algunos casos se convierte en un elemento imprescindible para poder acceder a otro tipo de recursos o ayudas. Y dentro de esta formación, lógicamente unida a la reciente llegada de un importante número de estos jóvenes, está la cuestión del aprendizaje del idioma, que en años anteriores había pasado a un segundo plano y que en la actualidad toma fuerza de nuevo.

El perfil que necesita todavía aprender castellano ha vuelto a estar presente cuando, de alguna forma, este perfil en los últimos años había desaparecido. (E 6)

Lo que últimamente estamos viendo es que la gente demanda cursos de castellano y que no hay sitio en ningún lado. (E 8)

Hoy por hoy si una persona viene a mi despacho y le derivo a las cinco, seis o siete organizaciones que dan clases de castellano, no le van a dar clase en ninguna. Y hasta que tú no enganches con una organización, no puedes empezar un itinerario. (E 6)

Sin embargo, las propias características del proceso de incorporación y sus dificultades hacen que el mantenimiento en dichos procesos no siempre sea fácil. Hay cuestiones como el no acceso a ayudas sociales, la saturación de algunos recursos o la obligación de pernoctar en la calle que hacen que difícilmente se pueda seguir adelante con un proceso de inserción más o menos normalizado.

Son procesos más largos y cada vez más complicados [...]. Prevemos que los procesos sean más largos. (E 4)

Pero si antes era complicado, ahora es el triple o más. Es que mira, necesitas tres años para conseguir la Renta de Garantía de Ingresos. (E 3)

Y puede llevarte a abandonar ese proceso del que estamos hablando, que es poder dormir todas las noches en una cama en el mundo albergue. $Y$ acabas diciendo que a la mierda con todo. (E 8)
Todo ello lleva a situaciones de frustración y desmotivación dentro de estos jóvenes, que en algunos casos acaban derivando en situaciones de deterioro personal, ya sea por la irrupción de patologías mentales y/o el uso y abuso de diferentes consumos.

La mayoría están como resignados. Y en otros vemos falta de control de impulsos. Y también vemos a otros con un problema de salud mental ya grave. Empiezan a consumir disolventes y pegamentos y con eso el deterioro en cuatro días es terrible [...]. Personas que en el inicio del proceso no tienen problemáticas añadidas, pero que terminan teniéndolas, debido al proceso de calle. (E 7)

Aparecen problemas de salud mental, consumos, ludopatía, etc. (E 12)

En conclusión, nos encontramos ante un colectivo que no muestra, en principio, una situación de exclusión social clásica y que más bien demanda la atención a través de recursos económicos o habitacionales. Sin embargo, la carencia de estos recursos hace que en algunos casos sí que acaben sufriendo situaciones de vulnerabilidad extrema e incluso exclusión social. Esta dinámica nos lleva, precisamente, a analizar cuáles son los recursos existentes y cómo están respondiendo a las necesidades de este colectivo concreto.

\section{4. (Más) necesidades y (menos) recursos: desbordamiento de la atención}

En lo referente a la atención a las personas sin hogar y los recursos existentes se dan dos dinámicas paralelas que confluyen en el momento actual. Por un lado, se viene detectando ya desde hace años que los jóvenes extranjeros que utilizan los recursos para personas sin hogar lo hacen porque no tienen ningún otro, pero no porque sea un colectivo que a priori tuviera que utilizarlos. Las debilidades de otros subsistemas de protección hacen que estos jóvenes tengan que acabar utilizando dichos recursos como una última opción, pero no están pensados para dar respuesta a las necesidades de dicho colectivo, con el riesgo de que acaben cronificando su situación e incluso empeorándola.

Aquí hay muchos sistemas que tiene algo que decir. [...] Porque nuestra premisa es que estos chavales con una Renta de Garantía de Ingresos y con una vivienda están funcionando. (E 12)

Yo creo que el tema de las viviendas comunitarias podría ser. [...] Buscar alternativas a los albergues. (E 1)

La mayoría de los que estamos recogiendo son los vacíos de otros sistemas; es que esos sistemas no se han desarrollado adecuadamente. $Y$ nosotros no estamos lo suficientemente especializados 
como para da una respuesta adecuada a esas personas. (E 3)

Lo que tenemos claro es que un chaval de estos no pinta nada en un centro de alojamiento nocturno. El que no tenga un problema muy gordo, en tres años lo va a tener. $Y$ entonces ya no vamos a tener ninguna duda de que es perfil específico de calle. (E 8)

Los educadores nos dicen que por favor saquemos a esos niños de allí [el albergue], que no están más que aprendiendo cosas que no les sirven de mucho... (E 9).

Por el otro lado, junto a este contexto de carácter más estructural, nos aparece otro coyuntural y que ya hemos analizado, el aumento de la llegada de menas a los centros tutelados, que hace que la presión sobre los recursos aumente considerablemente, más aún cuando estos se habían rediseñados en un contexto de parón en la llegada de menores.

Los programas de emancipación que existían para los exmenas tutelados se redujeron en el periodo de recesión, ya que no eran necesarias tantas plazas. Pero, en el momento actual, con un incremento tan grande de exmenas son programas que no son capaces de dar respuesta a todas las personas que cumplen los dieciocho años y dejan de estar tuteladas por ley. Esta saturación en los recursos de emancipación deviene en una saturación asimismo de los recursos para personas sin hogar.

Desde enero [de 2017] nos dimos cuenta. Oye, que están viniendo y nos va a pillar el toro. [...] Hay un desborde de la situación. (E2)

Los recursos de hoy en día no dan abasto. [...] Lo que nos pasa es que tenemos tantas demandas que hemos generado una lista de espera, tenemos una lista de espera de unos veinte chavales y los centros de menores nos dicen que en marzo salen otros veinte. (E 6)

De esta forma, el tránsito de mena a JENA aparece como un momento crítico en el que para gran parte de estos jóvenes se da una alta vulnerabilidad en esta transición de la tutela del menor a la emancipación del mayor de edad. Se pasa de un sistema de protección muy estable y consolidado a otro en el que el riesgo de exclusión aumenta considerablemente. Es por esto por lo que que gran parte de las personas entrevistadas subrayan la necesidad de facilitar dicha transición y de ofrecer recursos para el sostenimiento de estos procesos de incorporación social.

Si eres mena estás en inclusión; pero si eres JENA ya estás en exclusión. Y entonces ahí empieza todo este problema. (E 8)

Salgo con 18 años y... ¿ahora qué? Mi proyecto de vida y lo que estaba funcionando hasta ahora...
¿Cómo voy a seguir llevando mi proyecto en la calle? (E 1)

No puedes tener a un crío alojado dos o tres años, o los que sea, y luego que ahí termine el tema. Ahora va a haber un montón de mena, de chavales, que van a salir. ¿Y cuál es el plan? Pues el plan es que no hay plan. (E 8)

Por lo tanto, lo que se observa es que se entrecruzan dinámicas que venían de antes, como por ejemplo la falta de acomodo entre los recursos existentes y las personas jóvenes extranjeras, que podrían acceder a otros tipos de recursos pero que como no existen o no pueden acceder a ellos acaban utilizando los únicos disponibles. Pero, además, esta lógica se da en un contexto como el actual, en el que los recursos han mermado en un escenario de más necesidades, es decir, de más personas que atender. Como puede pensarse, el resultado de estas dos variables es el desbordamiento de los recursos y la incapacidad para dar respuesta a las necesidades del colectivo, sobre todo en el momento que cumplen la mayoría de edad.

Por último, vamos a situar la realidad de Bilbao en el conjunto estatal, para así poder ver hasta qué punto lo que ocurre en esta ciudad es algo exclusivo o si se trata, por el contrario, de una pauta más generalizada y que puede verse en otras ciudades. Para ello, preparamos un pequeño cuestionario que fue respondido por personal técnico de diferentes ciudades, como Valencia, Barcelona, A Coruña, Madrid, Gijón, Córdoba y Burgos. En este sentido, es interesante subrayar que se han detectado algunas pautas similares entre estas ciudades.

Así, sobre todo en las grandes, como es el caso de Madrid, Barcelona o A Coruña, se detecta un intenso incremento del número de personas sin hogar, sobre todo tras la recesión económica, y este aumento se debe también a la llegada de menas y JENA a estas ciudades, al igual que ocurre en el caso de Bilbao. Todo ello nos lleva a colegir que Bilbao no es una excepción y que muestra una pauta muy similar a otras grandes ciudades españolas.

\section{Conclusiones}

A lo largo de este artículo se han analizado las principales características del colectivo de las personas sin hogar residentes en Bilbao, haciendo especial hincapié en los cambios y las tendencias que se detectan en los últimos años y con respecto a periodos anteriores.

En este sentido, un primer rasgo a destacar es el considerable aumento del colectivo, debido en gran parte a un aumento en la llegada de los menas y una insuficiente red de recursos de emancipación, que hacen que al cumplir los dieciocho años un buen número de estos se vean abocados a tener que recurrir a los recursos existentes para el sinhogarismo. 
Así, y centrándonos en la cuestión de los perfiles, las personas jóvenes de origen magrebí se erigen en el grupo más relevante dentro de las personas sin hogar; ya lo eran en el periodo de la crisis económica, pero esta tendencia se acentúa intensamente en los últimos años. Como puede pensarse, el perfil de las personas sin hogar autóctono y más clásico tiende a perder fuerza. También irrumpen otros perfiles, como el de las mujeres, las familias o las personas solicitantes de asilo, pero en mucha menor cuantía.

Nos encontramos, de este modo, en un escenario en el que los recursos están mostrando dificultades para dar respuesta a este aumento del colectivo, más aún en un momento en el que algunos de estos recursos, como los dirigidos a la emancipación de los exmenas, habían sido redimensionados, ya que quedaban plazas vacantes en el periodo de recesión económica.

En un contexto de "más por menos", los recursos acaban sufriendo una especie de desbordamiento de carácter múltiple y secuencial: el aumento de la llegada de menas hace que la atención en los centros de menores no sea la idónea; los recursos de emancipación existentes no pueden atender a todos estos exmenas que abandonan los centros al cumplir los dieciocho años y el resultado final es que estos en gran parte tan solo pueden recurrir a los recursos existentes para personas sin hogar. En este escenario, el tránsito de mena a exmena se convierte en algo extremadamente complicado y traumático, en el que el proceso de inserción que había comenzado previamente puede zozobrar fácilmente.

Es cuanto menos una situación paradójica; personas que en principio no necesitan de un tipo de recursos muy concretos y para nada pensados y preparados para este colectivo, acaban utilizándolos y este propio uso acaba en no pocos casos creando situaciones de exclusión social y haciendo que finalmente sí que los necesiten utilizar. Dicho de otra manera, es la propia -inadecuada- atención, la que acaba haciendo que posteriormente acaben necesitando dicha atención y dichos recursos, en este caso los dirigidos a personas sin hogar.

De todo lo dicho hasta ahora, podría pensarse que una solución adecuada podría ser la de redimensionar de nuevo los recursos, ampliando aquellos que se vieron reducidos en años anteriores y estableciendo un sistema de emancipación para exmenas más amplio y generoso. Aunque en algún caso y para algún recurso se necesite una ampliación de la cobertura existente, no está nada claro que esta respuesta sea la idónea y adecuada.

El análisis de la evolución del número de personas sin hogar y del de menas apunta a amplias fluctuaciones y a dinámicas en muchos casos ajenas al ámbito de intervención de los servicios sociales, en el que se cruzan aspectos vinculados incluso a las relaciones internacionales y el estado de la relación entre países como España y Marruecos. En este sentido, intentar dar respuesta desde unos servicios sociales municipales o forales a situaciones que se sitúan en ámbitos superiores y que son completamente incontrolables por dichas instancias parece cuando menos algo arriesgado y poco eficaz. Dicho de otra forma, difícilmente va a poder darse una respuesta local a un tema que como poco es una cuestión de estado.

Además, no podemos olvidar que gran parte tanto de los menas como de los exmenas se dirigen a aquellas comunidades autónomas en las que se está dando una respuesta adecuada a dicha problemática, y en un contexto como este sería muy difícil poder dimensionar adecuadamente qué recursos son los suficientes y van a serlo a corto y medio plazo en una coyuntura de demanda infinita en la que siempre la atención va a ir por detrás de la demanda.

La mayoría de las personas entrevistadas, por ejemplo, creían que el aumento de la llegada de menas iba a ser constante e incesante en los próximos meses, pero los datos disponibles más recientes, sin embargo, no confirman esta hipótesis y parece que se detecta un importante parón en el arribo de este colectivo que, por lo tanto, influirá también en las magnitudes de exmenas que tengan que recurrir a los recursos para personas sin hogar.

Por ello, estimamos que habría que reflexionar sobre la búsqueda de un sistema eficaz y eficiente, más que en cómo ampliar los recursos ya existentes. En esta línea, es fundamental establecer un itinerario de emancipación, ajeno a los servicios sociales dirigidos para las personas sin hogar y en el que otros sistemas como el educativo o la vivienda tienen que jugar un papel fundamental. Hay que pensar en un modelo integral, que junto a estos sistemas también incluya un acompañamiento social y jurídico que pueda contribuir en los procesos de inserción. 
AIERDI et al. (2009): Perfil y necesidades de las personas sin hogar en Bizkaia, Bilbao, Diputación Foral de Bizkaia.

ARRIBA, A. (2002): "El concepto de exclusión en política social", Trabajo Social Hoy, 47-76.

BIZITEGI (2019): "Estudio sobre la realidad de las mujeres en situación de exclusión residencial”, Donostia-San Sebastián, Servicio Central de Publicaciones de Gobierno Vasco.

CASTELLS, M. (2003): “Internet, libertad y sociedad: una perspectiva analítica”, Polis, 4, 1-20.

FAINSTEIN, N. (1993): "Race, class and segregation: discourse about African Americans", International Journal of Urban and Regional Research, 384-403.

FUNDACIÓN FOESSA (2008): VI Informe sobre exclusión y desarrollo social en España 2008, Madrid, Caritas Española Editores.

HARRINGTON, M. (1984): The new American poverty, Holt, Rinehart and Winston.
LAPARRA, M. y PÉREZ, B. (2008): Exclusión social en España. Un espacio diverso y disperso en profunda transformación, Madrid, Fundación FOESSA.

MYRDAL, G. (1962): Challenge to affluence, Phanteon.

NAVARRO, M.A. (2018): Relatos de la calle: Testimonios de migrantes sin hogar, Madrid, Irredentos libros.

SUBIRATS, J. (2004): Pobreza y exclusión social. Un análisis de la realidad española y europea. Colección Estudios Sociales, 16.

VALLES, M. (2003): Técnicas cualitativas de investigación social, Madrid, Síntesis.

VILAGRASA, J. (2000): "Los debates sobre pobreza urbana y segregación social en Estados Unidos", Scripta Nova. Revista Electrónica de Geografía y Ciencias Sociales, 76.

WILSON, W.J. (1987): The Truly Disadvantage: The Inner City, the Undeclass, and Public Policy, University of Chicago Press. 